\title{
Soft Biometrics for Subject Identification using Clothing Attributes
}

\author{
Emad Sami Jaha*† \\ *Faculty of Computing and Information Technology \\ King Abdulaziz University \\ Jeddah, Saudi Arabia \\ ejaha@kau.edu.sa
}

\author{
Mark S. Nixon ${ }^{\dagger}$ \\ ${ }^{\dagger}$ School of Electronics and Computer Science \\ University of Southampton \\ Southampton, United Kingdom \\ msn@ecs.soton.ac.uk
}

\begin{abstract}
Recently, soft biometrics has emerged as a novel attribute-based person description for identification. It is likely that soft biometrics can be deployed where other biometrics cannot, and have stronger invariance properties than vision-based biometrics, such as invariance to illumination and contrast. Previously, a variety of bodily soft biometrics has been used for identifying people. Describing a person by their clothing properties is a natural task performed by people. As yet, clothing descriptions have attracted little attention for identification purposes. There has been some usage of clothing attributes to augment biometric description, but a detailed description has yet to be used. We show here how clothing traits can be exploited for identification purposes. We explore the validity and usability of a set of proposed semantic attributes. Human identification is performed, evaluated and compared using different proposed forms of soft clothing traits in addition and in isolation.
\end{abstract}

\section{Introduction}

Identifying people is an important task in daily life. Classic physical and behavioral biometrics have been widely and effectively used for person identification. The emergence of soft biometrics introduces a new form of biometric trait for identification. Soft biometrics use conventional human descriptions and translates them to the machine's biometric forms (in a way, bridging the semantic gap) [1]. Soft biometric techniques mainly depend on defining a number of semantic attributes and assigning a set of descriptive labels (traits) for each attribute. A semantic attribute can be any observable property that has a designated name or description by humans. Such attributes can either be binary attributes associated with categorical traits or relative attributes, which can be associated with categorical or comparative labels. So far, the relative descriptions have been found to be more precise and informative compared with the binary descriptions [2].

Despite the lack of discriminatory capability associated single soft traits, combination with other soft traits can be used as biometric signature for identification [3] or they can be used to augment other traditional physical and behavioral (hard) biometrics such as facial traits [4] or a gait signature [1]. Relative attributes are not only measurable, representing the strength of attributes, but also comparable allowing for more precise differentiation [2], emphasizing the small differences in attributes of one subject in comparison with those of others [3].

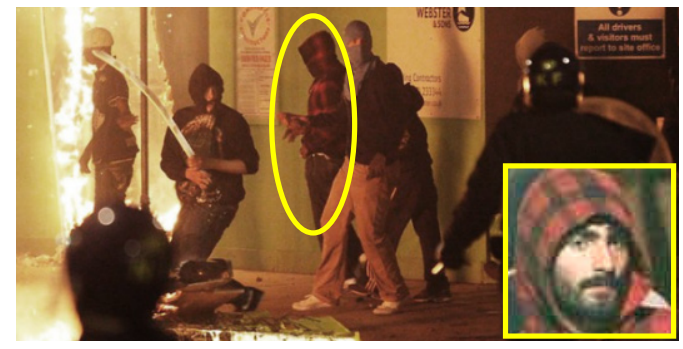

Figure 1: An image highlighting a marked suspect with covered face and distinct clothing ${ }^{1}$ and bottom right a face image of a suspect appearing to wear the same clothes ${ }^{2}$.

Although human clothes are a predominant visible characteristic of the person's appearance, they have yet to be adopted for representing soft biometric traits for an individual and have been considered unlikely as a cue to identity. Detecting the presence of some common clothing attributes, besides other soft biometrics, can supplement the low-level features used for person re-identification [5]. This allows use of more of the information available in surveillance video which is consistent with analyzing data of such poor quality.

Other than biometrics, there has been previous work on recognizing clothing categories [6], semantically describing clothing pieces [7] and automatically detecting then classifying certain semantic clothing attributes in pedestrian data images [8], or alternatively classifying overall clothing styles in natural scenes based on a group of defined common categories and attributes [9]. Also automatic search and retrieval by clothing attributes for occasion-style recommendation [10] or a bodily attribute-based query integrated with clothing colors and types for a people search in surveillance data [11].

In everyday life, people use clothing descriptions to

\footnotetext{
${ }^{1}$ The Advertiser: http://www.adelaidenow.com.au/news/world/profiles-oflondon-locations-where-riots-have-broken-out/story-e6frea81-1226111443592

${ }^{2}$ BBC News: http://www.bbc.co.uk/news/uk-england-london-16171972
} 
Table 1: Semantic clothing attributes and corresponding categorical and comparative labels used for annotation.

\begin{tabular}{|c|c|c|c|}
\hline Body zone & Semantic Attribute & Categorical Labels & Comparative Labels \\
\hline \multirow{4}{*}{ Head } & 1. Head clothing category & [None, Hat, Scarf, Mask, Cap] & \\
\hline & 2. Head coverage & [None, Slight, Fair, Most, All] & [Much Less, Less, Same, More, Much more] \\
\hline & 3. Face covered & [Yes, No, Don't know] & [Much Less, Less, Same, More, Much more] \\
\hline & 4. Hat & [Yes, No, Don't know] & \\
\hline \multirow{3}{*}{ Upper body } & $\begin{array}{l}\text { 5. Upper body clothing category } \\
\text { 6. Neckline shape }\end{array}$ & $\begin{array}{l}\text { [Jacket, Jumper, T-shirt, Shirt, Blouse, Sweater, Coat, Other] } \\
\text { [Strapless, V-shape, Round, Shirt collar, Don't know] }\end{array}$ & \\
\hline & 7. Neckline size & [Very Small, Small, Medium, Large, Very Large] & [Much Smaller, Smaller, Same, Larger, Much Larger] \\
\hline & 8. Sleeve length & [Very Short, Short, Medium, Long, Very Long] & [Much Shorter, Shorter, Same, Longer, Much Longer] \\
\hline \multirow{3}{*}{ Lower body } & $\begin{array}{l}\text { 9. Lower body clothing category } \\
\text { 10. Shape }\end{array}$ & \begin{tabular}{|l} 
[Trouser, Skirt, Dress] \\
[Straight, Skinny, Wide, Tight, Loose]
\end{tabular} & \\
\hline & 11. Leg length (of lower clothing) & [Very Short, Short, Medium, Long, Very Long] & [Much Shorter, Shorter, Same, Longer, Much Longer] \\
\hline & 12. Belt presence & [Yes, No, Don't know] & \\
\hline \multirow{2}{*}{ Foot } & 13. Shoes category & [Heels, Flip flops, Boot, Trainer, Shoe] & \\
\hline & 14. Heel level & [Flat/low, Medium, High, Very high] & [Much Lower, Lower, Same, Higher, Much higher] \\
\hline \multirow{5}{*}{$\begin{array}{l}\text { Attached to } \\
\text { body }\end{array}$} & 15. Attached object category & [None, Bag, Gun, Object in hand, gloves] & \\
\hline & 16. Bag (size) & [None, Side-bag, Cross-bag, Handbag, Backpack, Satchel] & [Much Smaller, Smaller, Same, Larger, Much Larger] \\
\hline & 17. Gun & [Yes, No, Don't know] & \\
\hline & 18. Object in hand & [Yes, No, Don't know] & \\
\hline & 19. Gloves & [Yes, No, Don't know] & \\
\hline General style & 20. Style category & $\begin{array}{l}\text { [Well-dressed, Business, Sporty, Fashionable, Casual, Nerd, } \\
\text { Bibes, Hippy, Religious, Gangsta, Tramp, Other] }\end{array}$ & \\
\hline Permanent & 21. Tattoos & [Yes, No, Don't know] & \\
\hline
\end{tabular}

identify/re-identify each other, especially from a distance or when the faces are not visible. Furthermore, many people often wear similar clothing or a certain clothing style. It has been shown that clothing attributes are naturally correlated and mutually dependent on each other [7]; this can be exploited when composing a biometric signature and even further to possibly infer some unknown attributes from the known ones. Fig. 1 shows an image of rioters in London 2011 and it highlights a suspect with covered face and head. No soft traits are observable except clothing attributes. Also in the bottom right corner, a CCTV image released later within a list of most wanted suspects shows clearly what appears to be the same clothing, suggesting an identification link to the rioter. Such an image provides a real example of how clothing attributes could be beneficial in identification and also demonstrates that, in some cases, clothing attributes can be the only observable soft traits to be exploited.

In this paper, we employ semantic clothing attributes as soft biometric traits for human identification and explore the validity and efficiency of clothing descriptions for identification. The main contributions of this paper are:

- new identification techniques using a combination of soft clothing-based biometrics in fusion and in isolation;

- increased discriminatory comparative traits derived using a Ranking SVM for clothing attributes;

- a new web-based mechanism for obtaining and analyzing human clothing labels and comparisons;

- performance assessment and comparison between different forms of soft clothing traits; and

- an investigation of clothing attributes viability and correlations.

Section 2 introduces the proposed semantic attributes and descriptive labels. Sections 3 and 4 describe the experimental methodology used in this research. Using soft clothing traits for human identification is demonstrated to supplement traditional approaches and alone in Sections 5 and 6 before concluding in Section 7.

\section{Clothing Attributes}

There are many possible clothing attributes and labels; an initial set of those which appear to be basic is studied in this research. Based on a number of general considerations, a set of attributes and their labels is chosen and defined so as to construct a feature vector. Firstly, this set of defined attributes is intended to be structural and comprehensive rather than a detailed description of clothing. The set is also suited to analysis of surveillance data, which is often vulnerable to challenges and obstacles such as low resolution, long distance, lighting conditions, pose and occlusion $[1,3,4,7,11,15]$. Secondly, the attributes are desired to be as understandable as possible, reliable, visible, and observable, allowing an annotator to describe what is being seen not what is being inferred. By taking these considerations into account a number of attributes deemed suitable for later investigation were excluded. Here we propose a set of semantic clothing attributes described with categorical and comparative labels.

\subsection{Categorical Clothing Labels}

Categorical labels can be defined as nameable descriptions used to describe semantic attributes of an individual's clothing, usually associated with multiple clothing categories or styles such as (Upper body clothing category: 'Jacket', 'Jumper', 'T-shirt' etc.) or can be labels describing the degree of presence of relative attributes such as (Sleeve length: 'Very short', 'Short', 'Medium' etc.) 


\subsection{Comparative Clothing Labels}

Comparative labels are nameable descriptions used to describe only relative attributes of an individual's clothing compared with another individual's clothing. In other words, these labels describe the degree of comparisons of relative attributes, such as (Neckline size: 'Much smaller', 'Smaller', 'Same', 'Larger' and 'Much larger').

A list of 21 semantic attributes is proposed and for each attribute a suitable group of categorical labels is specified to be used for describing these attributes. Furthermore, seven of the aforementioned 21 attributes are both categorical and relative, whereas the remaining 14 are unsuited for comparison because they are binary or multi-class attributes that can be described using only categorical (absolute) labels. Thus, the categorical labels of these seven relative attributes are extended to their corresponding comparative labels. Table 1 shows the list of proposed semantic attributes with their assigned categorical and comparative labels, where the seven relative comparable attributes are in bold. Each categorical label, which is designated for a non-relative attribute, is assigned an integer value as a numeric representation for its textual expression. Previously a practical psychological system [12] using bipolar scales to define traits was performed as a unique analysis on whole-body descriptions. We utilize this validated practical mechanism to better reflect the degree-of-strength of the relative attributes. Each of the seven relative attributes is formulated as a bipolar five-point scale. We assign a set of ordered categorical labels ranging from 1 to 5 and a set of comparative labels is assigned accordingly ranging from -2 to 2 . For all binary-label clothing attributes, such as 'Belt presence', we add a label 'Don't know' as a choice, which often can be a possible reasonable answer in real-life, reflecting the user's uncertainty as to whether an attribute is present or not.

\section{Methodology}

\subsection{Human Clothing Dataset}

The Soton Gait Database [13] is a standard database used for this research, as it comprises a subset of full-body frontal and side view still images of the persons for which body and face descriptions are already available. The front view images are used to collect the clothing descriptions. This subset consists of 115 individuals with a total of 128 frontal samples. Each sample of each individual is handled, throughout all the steps of this experiment, as an independent individual. That is due to the fact that all individuals with multiple samples are wearing different clothing in each sample. Hence, multiple samples of a single individual are considered as different and independent entities by which each entity represents that individual if wearing exactly the same clothing. Otherwise, it is considered as another entity, even though it belongs to the same individual, but wearing different clothing. In this research we refer to each entity (i.e. sample in this dataset) as a subject. $90 \%$ of the subjects in the database are university students and wear what is largely similar (summer) clothing (jeans, T-shirt, etc.). As such, the data appears sufficiently challenging for this initial study.

\subsection{Clothing Description Database}

A web-based clothing annotation system was designed and developed to obtain clothing labels and comparisons, as shown in Fig. 2. The annotation procedure was split into two tasks. The first task required a user to annotate ten subjects. Each subject was described by selecting 21 appropriate categorical labels. The second task required a user to compare one subject, selected randomly from the ten already annotated, with other ten new subjects. A comparison, between two subjects was performed by selecting seven appropriate comparative labels. In this way $M$ labelers provided $N$ labels on $O$ subjects. With a view to simplifying and organizing user annotations, the clothing attributes were grouped based on their zones and relevance as follows: Head, Upper body, Lower body, Foot, Attached to body, General style and Permanent as shown in Table 1, which was similarly used to view attributes and labels in annotation forms as illustrated in Fig. 2.

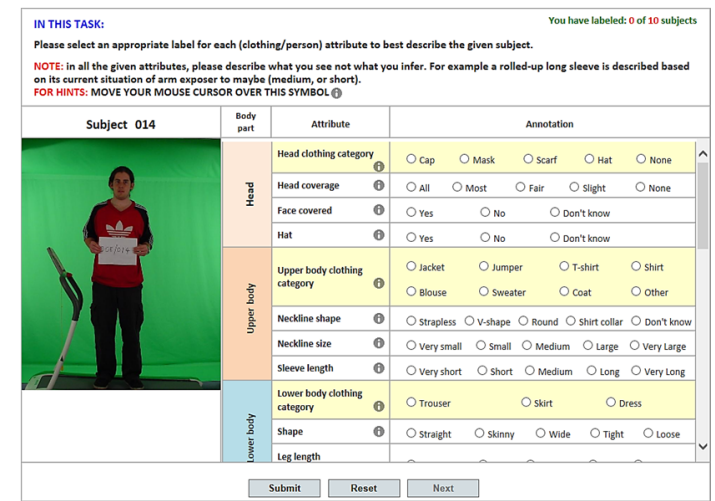

Figure 2: The website developed to obtain annotation data.

Table 2: The number of collected and inferred user data.

\begin{tabular}{|l|l|l|}
\hline Data summary & Collected & Inferred \\
\hline Total user annotations & 301 & N/A \\
\hline Total user comparisons & 307 & 533 \\
\hline Total attribute annotations & 6321 & N/A \\
\hline Total attribute comparisons & 2149 & 3731 \\
\hline Average user annotations per subject & 2.35 & N/A \\
\hline Average user comparisons per subject & 2.39 & 4.16 \\
\hline
\end{tabular}

Categorical and comparative labels were collected from 24 users via the website. All 128 samples were labeled by multiple users, with one or more separate user annotations per subject describing the 21 categorical attributes. All subjects were compared using the seven relative attributes by multiple users. To enrich the comparison data from the available number of collected comparisons, additional comparisons were inferred when two subjects were both compared with another same subject. A summary of 
collected user data and the inferred comparisons data is shown in Table 2.

\subsection{Ranking Relative Clothing Attributes}

The attribute-based clothing comparison data cannot be used for identification, unless it is arranged as a list of subjects ordered with respect to a single attribute. Subsequently, the attributes of each subject can be described by relative measurements derived from per attribute ordering data. Different ranking methods can be applied for this purpose, such as the Elo ranking system [3] or a Ranking SVM [2]. In this research, to achieve ordering and to derive the desired relative measurements, a soft-margin Ranking SVM method [14] is used, along with a supporting formulation of similarity constraints [2]. This is done to apply a pairwise technique based on learning a ranking function per attribute. Such learned ranking functions can be used not only to perceive the relative strength of attributes in a training sample, but also to predict the relative strength in a new test sample. Thus, for a set of attributes $A$, a ranking linear function $r_{a}$ is learned for each attribute $a$ such that:

$$
r_{a}\left(x_{i}\right)=w_{a}^{T} x_{i}
$$

where $w_{a}$ is the coefficient of the ranking function $r_{a}$ and $x_{i}$ is a feature vector of attributes of a subject being ranked. A set of comparisons is rearranged into two groups to represent the pairwise relative constraints required to learn a ranking function. The first group consists of a set of dissimilarity comparisons $D_{a}$ of ordered pairs so that $(i, j) \in$ $D_{a} \Rightarrow i>j$ whereas the second group comprises a set of similarity comparisons $S_{a}$ of non-ordered pairs so that $(i, j)$ $\in S_{a} \Rightarrow i=j . D_{a}$ and $S_{a}$ sets are then utilized to derive the $w_{a}$ coefficients of $r_{a}$ according to the following formulation:

$$
\begin{array}{lll}
\operatorname{minimize} & \left(\frac{1}{2}\left\|w_{a}^{T}\right\|^{2}+C \sum \xi_{i j}^{2}\right) & \\
\text { subject to } & w_{a}^{T}\left(x_{i}-x_{j}\right) \geq 1-\xi_{i j} ; \quad \forall(i, j) \in D_{a} \\
& \left|w_{a}^{T}\left(x_{i}-x_{j}\right)\right| \leq \xi_{i j} ; \quad \forall(i, j) \in S_{a} \\
& \xi_{i j} \geq 0
\end{array}
$$

The degree of misclassification is measured by $\xi_{i j}$ and the trade-off between maximizing the margin and minimizing the error (i.e. satisfying constraints) is denoted as $C$. The resulting optimal $w_{a}$ function is able to enforce (explicitly) a desirable ordering for all training samples, in respect to $a$. A feature vector $x_{i}$ is mapped using Equation (1) to a corresponding feature vector comprising a number of real-value relative measurements. Each measurement represents the relative strength of a single attribute.

\subsection{Soft Clothing Traits}

Categorical labels are used to form two feature vectors for each subject in the dataset. In both versions of feature vectors, an average label per attribute is calculated for a set of labels provided by multiple users describing the same subject. The first feature vector is formed from 21 categorical normalized average-labels, describing all the 21 clothing attributes to build the first gallery of categorical clothing traits for all subjects (referred to as Cat-21). Similarly, the second feature vector is formed from a subset of only the seven relative clothing attributes to build the second gallery (referred to as Cat-7).

To perform identification using comparative labels, the corresponding relative measurements are derived using the Ranking SVM method. All subjects in the Cat-7 gallery are used as a training dataset to learn seven optimal ranking functions for the seven relative attributes. The coefficient $w$ is derived using the formulation in Eqn. (2). The desirable per attribute ordering of all subjects is derived using $w$. Then by Eqn. (1), each $w$ is used to map each feature vector in Cat-7 to a corresponding vector of seven relative measurements (i.e. comparative traits) describing a single subject. Hence, all the obtained relative measurement vectors are gathered to compose a new gallery of comparative traits (referred to as $\mathrm{Cmp}$ ).

\subsection{Performance Evaluation}

Leave-one-out testing is used to evaluate the clothing traits performance. Thereby, user annotations are used in turn as a query to identify and retrieve matching subjects from a tested gallery. Hence, two Cat- $N$ based probe sets are designed such that, each subject's annotation provided by a single annotator, is normalized and formulated as 21-label and 7-label feature vectors to be used to probe Cat-21 and Cat-7 galleries. The same 7-label probe set used for testing Cat-7 is used to probe the Cmp gallery, except that each feature vector in the probe set is mapped to its comparative-traits vector predicted by $w$. This mapping is performed to produce the third Cmp-based probe set containing a new form of testing feature vector that is able to probe the Cmp gallery. The likelihood between every single probe-vector and all subject-vectors in a gallery is estimated and retrieved, resulting in an ordered list of all subjects based on the likelihood evaluated by the sum of Euclidean distance between probe and gallery vectors. The Cumulative Match Characteristic (CMC) and Receiver Operator Characteristic (ROC) are used to summarize identification performance, via the $k$ nearest neighbor approach. The Decidability Index $\left(d^{\prime}\right)$ of the normalized distance between the two means of genuine $G$ and imposter $I$ distributions such that $d^{\prime}=\left|\mu_{G}-\mu_{I}\right| / \sqrt{\left(\sigma_{G}^{2}+\sigma_{I}^{2}\right) / 2}$.

\section{Correlation between Attributes}

We study the proposed clothing attributes' relationships using the (Pearson's $r$ ) correlation matrix. We generated correlation matrices for the available collected labels, comparisons and further derived traits. The calculated correlation coefficient was considered as significant when 
the resulting $p$-value $p \leq 0.05$. Fig. 3 demonstrates the correlation between all labels of 18 clothing attributes (see Table 1); traits without correlation are not shown. High correlation is symbolized by orange, and low by blue/ green. As such, attributes relating to head coverage are highly correlated, as are the attributes (15) and (18) relating to the description of items attached to the body. Clothing is well correlated for upper (5) and lower (9) body. The matrix structure suggests that the desired uniqueness has been achieved.

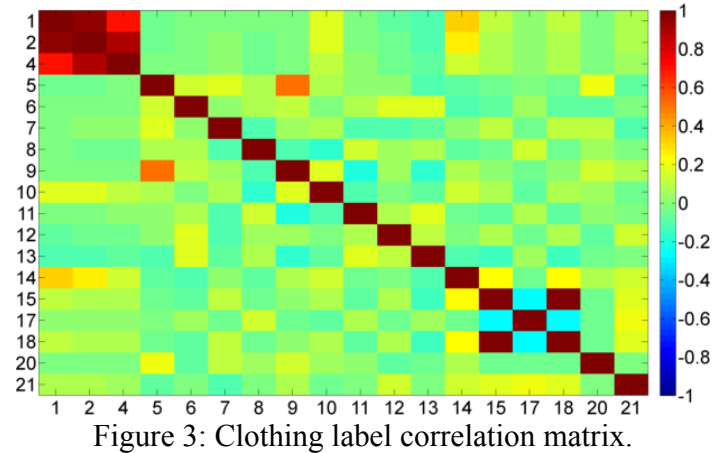

\section{Augmenting Soft Biometrics with Clothing}

Previously, soft biometrics body descriptions were obtained from the Soton database, in which each of 115 individuals were labeled by multiple users' annotations describing 23 soft bodily traits. These traits were grouped into three categories: Body shape, Global, and Head [1].

We use our three proposed approaches (Cat-21, Cat-7 and Cmp) of soft clothing traits to enhance the identification performance in two different aspects. The first aspect is to augment only the traditional soft traits (Age, Ethnicity, Sex, and Skin Color) which were the four traits grouped in the Global category in [1]. The second aspect is to augment all the aforementioned 23 soft body traits including the four traditional traits.

For each subject we form a single 23-trait feature vector from the multiple annotations of that subject to build a gallery of soft bodily descriptions for all subjects (referred to as $s o f t B o d y$ ). From softBody we derive another gallery of a traditional four-trait feature vector per subject (referred to as tradSoft) comprising only traditional soft descriptions.

Both tradSoft and softBody are extended to three other versions supplemented by the proposed soft clothing traits. Each feature vector describing a single subject in tradBody is concatenated to a corresponding feature vector describing the same subject in each of Cat-21, Cat-7 and Cmp to compose three versions of augmented galleries: tradCat-21, tradCat-7 and tradCmp respectively. The same process is applied to softBody, resulting in its three augmented versions softCat-21, softCat-7 and softCmp. Each softBody and tradSoft is tested for identification separately and then compared to its three augmented versions. For testing, proper probe sets are prepared for
softBody and tradSoft, so that for each subject we select a number of annotations from body-descriptions data equal to the number of annotations collected for clothing descriptions. These probe sets are extended to their corresponding augmented versions, according to their galleries, by concatenating them to each of the three clothing probe sets to enable the testing of their augmented galleries. Based on the leave-one-out method, human identification using bodily soft approaches and their augmented counterparts is achieved and evaluated.

\subsection{Augmenting Traditional Soft Biometrics}

Table 3 reports the $\mathrm{CMC}$ match scores and average-sum scores in different ranks for the traditional soft traits (Age, Ethnicity, Sex, and Skin Color) and when augmented by clothing. Adding clothing to traditional soft biometrics consistently enhances identification with up to $59 \%$ offered by the categorical traits tradCat-21, which achieves the highest scores in all ranks. tradCat-7 comes next in performance, then tradCmp with slightly lower scores. Fig. 4 shows ROC curves of the augmenting approaches have better accuracy and less error, where tradCat-21 consistently presents the highest accuracy, followed by tradCat-7. While Table 3 indicates the smallest Equal Error Rate (EER) and the Area Under the ROC Curve (AUC) for tradCat-21, tradCmp receives the best score in the decidability metric $d^{\prime}$; all the best values are shown in bold.

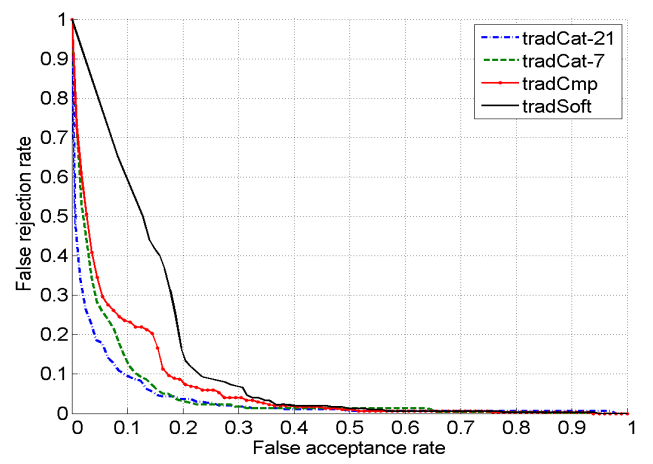

Figure 4: ROC performance of traditional soft biometrics and when augmented by clothing.

Table 3: CMC match scores, and ROC metrics of traditional soft biometrics and when augmented by clothing.

\begin{tabular}{|l|c|c|c|c||c|c|c|}
\hline \multirow{2}{*}{ Approach } & $\begin{array}{c}\text { Top } \\
\text { rank }\end{array}$ & \multicolumn{2}{|c||}{$\begin{array}{c}\text { AVG sum match } \\
\text { scores up to rank }\end{array}$} & $\begin{array}{c}\mathbf{1 0 0 \%} \text { accuracy } \\
\text { achieved at rank }\end{array}$ & EER & AUC & $\boldsymbol{d}^{\prime}$ \\
\cline { 2 - 9 } & $=\mathbf{1}$ & $\mathbf{1 0}$ & $=\mathbf{1 2 8}$ & & & & \\
\hline tradSoft & 0.16 & 0.31 & 0.866 & 72 & 0.181 & 0.136 & 1.734 \\
\hline tradCat -21 & $\mathbf{0 . 7 5}$ & $\mathbf{0 . 9 2}$ & $\mathbf{0 . 9 9 3}$ & $\mathbf{2 9}$ & $\mathbf{0 . 0 9 7}$ & $\mathbf{0 . 0 3 6}$ & 1.789 \\
\hline tradCat -7 & 0.47 & 0.76 & 0.974 & 37 & 0.108 & 0.050 & 2.010 \\
\hline tradCmp & 0.44 & 0.74 & 0.966 & 63 & 0.161 & 0.069 & $\mathbf{2 . 0 4 5}$ \\
\hline
\end{tabular}

\subsection{Augmenting Body Soft Biometrics}

The CMC curves in Fig. 5 and the results in Table 4 represent the identification performance using soft body traits with performance augmented by soft clothing traits. The performance of the body soft traits is considerably 
improved by adding the clothing yielding an increase ranging from $7 \%$ to $17 \%$ in the rank 1 result. The identification rate of softBody jumps from $78 \%$ to $95 \%$ when augmented by categorical traits softCat-21, and reaches $100 \%$ rapidly at rank 9 . Likewise, augmenting the traditional soft approach, softCat-7 remains, achieving the second best performance and the comparative measures softCmp holds the highest decidability $d^{\prime}$.

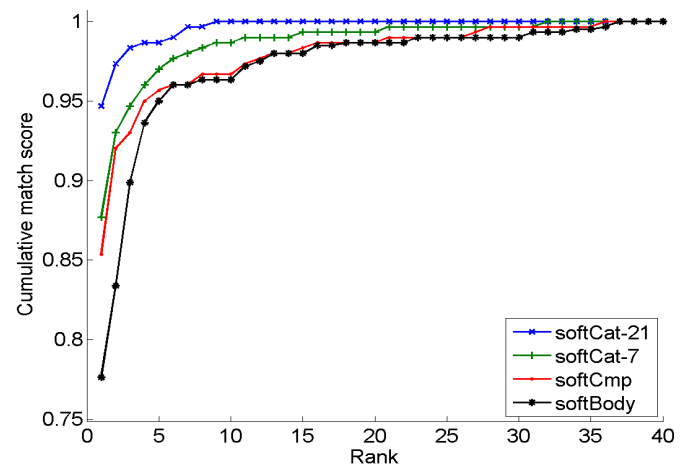

Figure 5: CMC performance (up to rank 40) of body soft biometrics and when augmented by clothing.

Table 4: CMC match scores, and ROC metrics of the body soft biometrics and when augmented by clothing.

\begin{tabular}{|l|c|c|c|c||c|c|c|}
\hline \multirow{2}{*}{ Approach } & $\begin{array}{c}\text { Top } \\
\text { rank }\end{array}$ & \multicolumn{2}{|c|}{$\begin{array}{c}\text { AVG sum match } \\
\text { scores up to rank }\end{array}$} & $\begin{array}{c}\mathbf{1 0 0 \%} \text { accuracy } \\
\text { achieved at rank }\end{array}$ & EER & AUC & \multirow{2}{*}{$\boldsymbol{d}^{\prime}$} \\
\cline { 2 - 9 } & $\mathbf{1}$ & $\mathbf{1 0}$ & $\mathbf{1 2 8}$ & & & & \\
\hline softBody & 0.78 & 0.92 & 0.991 & 37 & 0.087 & 0.028 & 2.785 \\
\hline softCat -21 & $\mathbf{0 . 9 5}$ & $\mathbf{0 . 9 9}$ & $\mathbf{0 . 9 9 9}$ & $\mathbf{9}$ & $\mathbf{0 . 0 5 0}$ & $\mathbf{0 . 0 1 4}$ & 2.634 \\
\hline softCat -7 & 0.88 & 0.96 & 0.996 & 32 & 0.063 & 0.018 & 2.814 \\
\hline softCmp & 0.85 & 0.94 & 0.994 & 36 & 0.080 & 0.026 & $\mathbf{2 . 8 2 7}$ \\
\hline
\end{tabular}

\section{Identification Using Soft Clothing Traits}

Each of the proposed approaches (Cat-21, Cat-7 and Cmp) is used in isolation for human identification, when soft clothing traits are the only used biometric. Here the leave-one-out method is also applied to probe the three soft clothing galleries using the appropriate probe set for each. The CMC scores and the ROC analysis of the proposed approaches are given in Table 5. Although all the approaches tend to improve the identification score sharply throughout the rank increase from 1 to 10 , the categorical labels Cat-21 start from a much better score and gain a much higher average score than Cat-7 and Cmp. The Cat-21 approach outperforms the other approaches in all terms, but the decidability $d^{\prime}$ is the largest and best in Cmp. It is noteworthy that, unlike in augmenting soft biometrics, the comparative traits $C m p$ achieve a better performance than their categorical counterparts underlying Cat-7.

Table 5: CMC match scores, and ROC metrics of clothing-based soft biometrics when used alone for identification.

\begin{tabular}{|l|c|c|c|c||c|c|c|}
\hline \multirow{2}{*}{ Approach } & $\begin{array}{c}\text { Top } \\
\text { rank }\end{array}$ & \multicolumn{2}{|c|}{$\begin{array}{c}\text { AVG sum match } \\
\text { scores up to rank }\end{array}$} & $\begin{array}{c}\text { 100\% accuracy } \\
\text { achieved at rank }\end{array}$ & EER & AUC & \multirow{2}{*}{$\boldsymbol{d}^{\prime}$} \\
\cline { 2 - 5 } & $=\mathbf{1}$ & $=\mathbf{1 0}$ & $\mathbf{= 1 2 8}$ & & & & \\
\hline Cat -21 & $\mathbf{0 . 6 3}$ & $\mathbf{0 . 8 4 3}$ & $\mathbf{0 . 9 8 4}$ & $\mathbf{4 1}$ & $\mathbf{0 . 1 3 7}$ & $\mathbf{0 . 0 5 9}$ & 1.442 \\
\hline Cat -7 & 0.27 & 0.507 & 0.923 & 92 & 0.192 & 0.108 & 1.303 \\
\hline Cmp & 0.28 & 0.510 & 0.929 & 96 & 0.174 & 0.088 & $\mathbf{1 . 8 2 4}$ \\
\hline
\end{tabular}

\section{Conclusions and Discussions}

Clothing characteristics can be utilized to convey effective descriptions and valuable combinations of soft biometrics. In some cases, human clothing descriptions might be the only observable attributes and a beneficial clue for identification. In our study, we find that the relative attributes underlying all the proposed clothing-based approaches carry the most significant and effective information to describe clothing. A good correlation between two attributes allows for the prediction of a missing attribute of those most likely from the other.

The obtained performance results using semantic clothing attributes indicate a motivation for exploiting derived clothing traits, in fusion or even in isolation, to enrich human identification and re-identification. The proposed techniques enforce and identification task by using clothing descriptions, in supplement to traditional or bodily soft traits, and as the only biometrics. We enable the implicit use of the rich correlations of clothing attributes. We show that, as a proof-of-concept, we can recognize people using soft clothing traits and we need further to learn a soft attribute-centric for retrieval in the future.

\section{References}

[1] S. Samangooei and M. S. Nixon, Performing content-based retrieval of humans using gait biometrics, Multimedia Tools and Applications, 2010.

[2] D. Parikh and K. Grauman, Relative attributes, in ICCV, 2011.

[3] D. Reid, M. Nixon, et al, Soft Biometrics; Human Identification using Comparative Descriptions, IEEE TPAMI, 2014.

[4] K. Niinuma, U. Park, and A. K. Jain, Soft biometric traits for continuous user authentication, IEEE Transactions on Information Forensics and Security, 2010.

[5] R. Layne, T. M. Hospedales, and S. Gong, Person Re-identification by Attributes, in Proc. BMVC, 2012.

[6] S. Liu, Z. Song, G. Liu, C. Xu, H. Lu, and S. Yan, Street-to-shop: Cross-scenario clothing retrieval via parts alignment and auxiliary set, in Proc. CVPR, 2012.

[7] H. Chen, A. Gallagher, and B. Girod, Describing clothing by semantic attributes, in ECCV, 2012.

[8] J. Zhu, S. Liao, Z. Lei, D. Yi, and S. Z. Li, Pedestrian Attribute Classification in Surveillance, in ICCV, 2013.

[9] L. Bossard, M. Dantone, C. Leistner, C. Wengert, T. Quack, and L. Van Gool, Apparel classification with style, in ACCV, 2013.

[10] S. Liu, J. Feng, Z. Song, T. Zhang, H. Lu, C. Xu, et al., Hi, magic closet, tell me what to wear!, in Proc. ACM MM12, 2012.

[11] D. A. Vaquero, R. S. Feris, D. Tran, L. Brown, A. Hampapur, and M. Turk, Attribute-based people search in surveillance environments, in WACV, 2009.

[12] M. D. MacLeod, J. N. Frowley, and J. W. Shepherd, Whole body information: its relevance to eyewitnesses, in Adult eyewitness testimony, ed: Cambridge Uni. Press, 1994.

[13] J. Shutler, M. Grant, M. S. Nixon, and J. N. Carter, On a large sequence-based human gait database, in RASC, 2002.

[14] T. Joachims, Optimizing search engines using clickthrough data, in Proc. ACM SIGKDD, 2002.

[15] D. Reid, S. Samangooei, C. Chen, M. Nixon, and A. Ross, Soft biometrics for surveillance: an overview, in Machine learning: theory and applications. Elsevier, 2013. 